\title{
The Unavailability of Preventive Care for Children during the COVID-19 Pandemic and Parental Mental Health
}

\begin{abstract}
The outbreak of the coronavirus disease 2019 (COVID-19) causing millions of people to be infected has posed major public health and governance challenges. This study evaluates the extent to which the unavailability of preventive care services for children due to the pandemic affects parental psychological wellbeing. We find that parents having no access to preventive care services for their children due to the pandemic are 6.55, 5.04, 3.90, and 3.85 percentage points more likely to feel anxious, worried, displeased, and depressed every day. The study calls for the expansion of preventive care services for children during the pandemic, especially for disproportionately affected communities.
\end{abstract}

